\title{
Impact of structured physical therapy intervention on functional recovery in a patient with CAPOS syndrome: a case report
}

\author{
Shailesh Gardas ${ }^{1 *}$ (1) and Aishwarya Mahajan²
}

\begin{abstract}
Background: CAPOS syndrome (cerebellar ataxia, areflexia, pescavus, optic atrophy, and sensorineural hearing loss) is a rare congenital autosomal dominant disorder. The resulting neurological sequelae of impairments are progressive in nature and may interfere with functional independence, performing activities of daily living (ADL's), and subsequently, affecting the quality of life (QOL). Since it is an extremely rare disorder, there is a severe dearth in the literature about how specific physiotherapy interventions may affect their functional status. Therefore, our objective was to investigate the effects of proprioceptive neuromuscular facilitation (PNF) and Frenkel's coordination exercises on functional recovery in a patient with CAPOS syndrome.
\end{abstract}

Case presentation: We herein present a case of a 25 -year-old Indian male with complaints of generalized body weakness, difficulty visualizing distant objects, nystagmus, progressive sensorineural deafness, and ataxia. He was rehabilitated with a structured/customized physiotherapy protocol consisting of PNF approach and coordination exercises for 4 weeks, 6 days/week, 60 min daily. An improvement in overall functional performance of patient as per postintervention scores of manual muscle testing, trunk control measurement scale, functional independence measure (components of self-care, transfers, and locomotion), and decline in severity of ataxia on scale for assessment and rating of ataxia scale was observed.

Conclusion: PNF and Frenkel's exercises resulted in an improvement in overall functional performance of the patient. Improvement was observed in post-test scores of Manual Muscle Testing (MMT), Trunk Control Measurement Scale (TCMS), and Functional Independence Measure (FIM) for the components of self-care, transfers, and locomotion. Additionally, results also showed a decline in severity of ataxia on post-test scores of scale for the assessment and rating of ataxia (SARA) scale (i.e., from severe to moderate).

Keywords: CAPOS syndrome, Proprioceptive neuromuscular facilitation, Frenkel's exercises, Case report

\section{Background}

Cerebellar ataxia, areflexia, pescavus, optic atrophy, and sensorineural hearing loss (CAPOS) syndrome was first reported in 1996 by Nicolaides et al. in a family with a maternally dominant inherited pattern [1]. It was found

\footnotetext{
* Correspondence: shaileshgardas@gmail.com

'Mahatma Gandhi Mission's College of Physiotherapy, Vashi, Navi Mumbai 400705, India

Full list of author information is available at the end of the article
}

to have an association with a mutation in the $A T P 1 A 3$ gene. Classically, patients have an onset from 6 months to 7 years and present with acute non-specific recurrent febrile sickness accompanied by persistent neurological symptoms like hypotonia, areflexia, nystagmus, strabismus, dysarthria, lethargy, and cerebellar ataxia. In addition, there are reports of a variable degree of optic atrophy, sensorineural hearing loss, and pes cavus seen in adolescence [2-4]. 
Proprioceptive neuromuscular facilitation (PNF) is one of the well-established treatment techniques incorporating functional diagonal movement patterns that helps facilitate, strengthen, and promote motor control in the rehabilitation of neurological disorders [5]. Previously, there has been documented evidence suggesting effectiveness of PNF trunk exercises on stability, balance, and upper limb function in patients with stroke. A study by Dionisio et al. revealed that RS during the task of reaching promotes an increase of multifidus activity ipsilateral to its application [6].

Evidence has proven effectiveness of a wide range of physical therapy (PT) interventions comprising of proprioceptive neuromuscular facilitation (PNF), Frenkel's exercises, dynamic postural stability training, muscle strengthening and flexibility exercises, balance reeducation, and gait training in patients with cerebellar disorders $[7,8]$. Out of the nine studies studied in a systematic review by Martin et al. [7] on investigating the effectiveness of physiotherapy in adults with cerebellar dysfunction; five studies used PNF and Frenkel's exercise in their intervention protocol. They found modest evidence that suggested physiotherapy has a positive effect on gait, trunk control, and activity limitations in people with cerebellar dysfunction. Additionally, several clinical trials and a case study conducted on individuals with ataxia have indicated a significant improvement in functional capabilities, such as self-care, gait, and in ataxic symptoms after rehabilitation programs [9-11].

However, to our knowledge, there is a serious gap in literature to determine the impact of rehabilitation in such a rare disease. Thus, the current case report was conducted to find out how a diagnosed case of CAPOS syndrome responds to PT intervention involving PNF and Frenkel's coordination exercises and thereby add value to present resources for rehabilitation of such rare cases.

\section{Case presentation \\ History}

Presenting a case of 25-year-old young Indian male, second child born at term out of a non-consanguineous marriage, achieved all typical milestones at the expected times and demonstrated a normal psychomotor development. In his early childhood, he experienced several episodes of non-specific febrile illness accompanied by generalized body weakness, clumsy bodily movements, and balance dysfunctions requiring frequent hospital admissions. With advancing age, he experienced a gradual decline in visualizing distant objects accompanied by a profound hearing loss more on right side. Presently, he is experiencing generalized body weakness, difficulty visualizing distant objects, nystagmus, progressive sensorineural deafness, incoordination in all four limbs, sitting balance dysfunction, truncal hypotonia, ataxia, and mild dysarthria. These primary neurological impairments have shown to interfere with independence to carry out activities of daily living (ADLs) such as sit to stand, independent standing, and walking which led to total dependence on caregivers. The relatives gave a positive family history in the patients elder sibling, who remained undiagnosed till he died at a young age of 18 years. Details of the same could not be retrieved from the family.

\section{PT assessment}

According to the initial neurological assessment, general condition was fair with an ectomorphic built with no history pre-existing systemic illnesses. Observational findings revealed bilaterally (b/l) dilated pupil, muscle wasting on all four limbs including intrinsic muscles of the hand and an appearance of plantar flexed, inverted cavus feet. Further on clinical examination, higher mental functions, attention, and memory were intact. Cranial nerve examination for $\mathrm{CN}$ II revealed a loss of distant vision on Snellen's chart. CN III indicated absence of pupillary constriction, while results of CN III, IV, and VI demonstrated nystagmus with abnormal saccadic eye movements and saccadic pursuits. CN VIII indicated sensorineural hearing loss on Weber's and Rinne's test. Sensory examination demonstrated intact superficial, deep, and combined cortical sensations. Non-equilibrium tests such as finger nose test, diadakokinesia, and heel shin revealed severe incoordination as seen by past pointing and intentional tremors. Also, sitting on the edge of the bed (equilibrium test) was not achievable without support suggesting severe affection. Muscle tone assessment indicated hypotonia, diminished deep tendon reflexes with b/l pendular knee jerk. Moderate muscle tightness was noted in $b / l$ hamstrings severe tightness in b/l plantar flexors. Muscle strength was assessed using Manual Muscle Testing (MMT) as per Medical Research Council (MRC) grading. Scale for the assessment and rating of ataxia (SARA) demonstrated moderate to severe ataxia. Trunk control measurement was performed using Trunk Control Measurement Scale (TCMS), findings of which indicated poor trunk control.

Tables 1 and 2 depict performance on outcome measures from baseline to post 4 weeks of intervention.

Physical and functional examination indicated involvement of cerebellum which was confirmed by MRI findings suggesting bilateral cerebellar atrophy. Computed topography of brain revealed loss of hair cells in the right mastoid. Additionally, audiometric report confirmed moderate sensorineural loss on (left) with an extensive loss on right with decreased hearing abilities. 
Table 1 Pre- and post-intervention scores of MMT

\begin{tabular}{|c|c|c|c|c|}
\hline \multirow[t]{2}{*}{$\mathrm{MMT}^{\mathrm{a}}$} & \multicolumn{2}{|c|}{ Pre-intervention (baseline) } & \multicolumn{2}{|c|}{ Post-intervention (post 4 weeks) } \\
\hline & Right & Left & Right & Left \\
\hline \multicolumn{5}{|l|}{ Shoulder } \\
\hline Flexors & $2 / 5$ & $2 / 5$ & $4 / 5$ & $4 / 5$ \\
\hline Extensors & $2 / 5$ & $1 / 5$ & $4 / 5$ & $3 / 5$ \\
\hline Abductors & $2 / 5$ & $2 / 5$ & $4 / 5$ & $4 / 5$ \\
\hline Rotators & $1 / 5$ & $1 / 5$ & $3 / 5$ & $3 / 5$ \\
\hline \multicolumn{5}{|l|}{ Elbow } \\
\hline Flexors & $2 / 5$ & $2 / 5$ & $4 / 5$ & $4 / 5$ \\
\hline Extensors & $2 / 5$ & $2 / 5$ & $3 / 5$ & $3 / 5$ \\
\hline \multicolumn{5}{|l|}{ Wrist } \\
\hline Flexors & $1 / 5$ & $1 / 5$ & $3 / 5$ & $3 / 5$ \\
\hline Extensors & $2 / 5$ & $2 / 5$ & $4 / 5$ & $4 / 5$ \\
\hline \multicolumn{5}{|l|}{ Hip } \\
\hline Flexors & $1 / 5$ & $1 / 5$ & $3 / 5$ & $3 / 5$ \\
\hline Extensors & $2 / 5$ & $2 / 5$ & $4 / 5$ & $4 / 5$ \\
\hline Abductors & $1 / 5$ & $1 / 5$ & $3 / 5$ & $3 / 5$ \\
\hline \multicolumn{5}{|l|}{ Knee } \\
\hline Flexors & $1 / 5$ & $1 / 5$ & $3 / 5$ & $3 / 5$ \\
\hline Extensors & $2 / 5$ & $2 / 5$ & $4 / 5$ & $4 / 5$ \\
\hline \multicolumn{5}{|l|}{ Ankle } \\
\hline Plantarflexors & $1 / 5$ & $1 / 5$ & $3 / 5$ & $3 / 5$ \\
\hline Dorsiflexors & $2 / 5$ & $2 / 5$ & $3 / 5$ & $3 / 5$ \\
\hline Invertors & $2 / 5$ & $2 / 5$ & $3 / 5$ & $3 / 5$ \\
\hline Evertors & $1 / 5$ & $1 / 5$ & $2 / 5$ & $2 / 5$ \\
\hline \multicolumn{5}{|l|}{ Trunk } \\
\hline Flexors & 2 & & 3 & \\
\hline Extensors & 2 & & 3 & \\
\hline
\end{tabular}

MMT Manual Muscle Testing

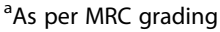

Table 2 Pre- and post-intervention score of Trunk Control Measurement Scale, Scale for the assessment and rating of ataxia, and functional independence measure scale

\begin{tabular}{lll}
\hline Outcome measures & $\begin{array}{l}\text { Pre-intervention } \\
\text { (baseline) }\end{array}$ & $\begin{array}{l}\text { Post-intervention } \\
\text { (post 4 weeks) }\end{array}$ \\
\hline TCMS & $17 / 58$ & $30 / 58$ \\
SARA & $35 / 40$ & $28 / 40$
\end{tabular}

FIM score

- Self-care $\quad 37 / 56 \quad 44 / 56$

- Transfers 6/21 13/21

- Locomotion 2/14 8/14

- Communication 9/14 9/14

- Social cognition 18/21 18/21

TCMS Trunk Control Measurement Scale, SARA Scale for the assessment and rating of ataxia, FIM Functional independence measure scale
Intervention goals and dosage

PT interventions were designed in order to improve balance and postural reactions in sitting as well as standing against external stimuli and gravity. Our aim was to improve joint stabilization by gaining good postural control both during independent sitting and standing in the long run. Also, treatment focused on promoting coordination in functional positions and patterns to achieve independence in ADL's. Total treatment dosage was once a day (6 days/week) over 4 weeks (total 24 sessions) period for 60 min with appropriate patient- and therapist-controlled rest intervals. Session started with $15 \mathrm{~min}$ of general warm-up exercises such as passive movements, stretching of lower limb muscles with active-assisted mobility of upper limb and trunk for 10 repetitions of two sets followed by $45 \mathrm{~min}$ of exercises involving PNF and Frenkel's respectively. Table 3 describes details of intervention protocol that was administered. During the 
Table 3 Details of intervention protocol that was administered

\begin{tabular}{|c|c|}
\hline Rationale & Treatment activities \\
\hline \multicolumn{2}{|l|}{ Week 1} \\
\hline To improve upper limb and trunk muscle strength & $\begin{array}{l}\text { Core stability: Static abdominals, partial curls, and trunk rotations. } \\
\text { PNF: Rhythmic initiation and active-assisted symmetrical UL and LL, D1, } \\
\text { and D2 flexion-extension along with pelvic and scapular patterns in } \\
\text { reclining positions in bed. } \\
\text { Stretching: B/L hamstrings, calves, invertors groups of muscles. }\end{array}$ \\
\hline $\begin{array}{l}\text { Promote limb coordination and gaze stabilization } \\
\text { (continued in every week) }\end{array}$ & $\begin{array}{l}\text { Frenkel's exercises: Fowler's position for ataxia in UL and LL movements } \\
\text { in PNF diagonal patterns using auditory and visual cues starting involving } \\
\text { single joint (assistance was provided if needed). } \\
\text { Eyes open, active eye and head movements between two stationary targets } \\
\text { as self-selected speed. }\end{array}$ \\
\hline \multicolumn{2}{|l|}{ Week 2} \\
\hline Promote upper limb muscle strength and coordination & PNF patterns progressed to active and bilateral patterns in Fowler's position. \\
\hline Improve postural control in sitting & RS and SR with minimal manual resistance in quadruped and bridge positions. \\
\hline Improve upper and lower limb coordination & $\begin{array}{l}\text { Frenkel's exercises in EOB position with minimal support (emphasizing on } \\
\text { multiple/combination joint movements in functional patterns). }\end{array}$ \\
\hline To improve strength of intrinsic muscles of hand & Exercises to improve strength of intrinsic muscles of hand were incorporated. \\
\hline \multicolumn{2}{|l|}{ Week 3} \\
\hline $\begin{array}{l}\text { Promote dynamic sitting control and anticipatory } \\
\text { postural reactions }\end{array}$ & $\begin{array}{l}\text { Sitting EOB: Starting with midline and then multidirectional reach outs under } \\
\text { supervision. } \\
\text { RS and SR with manual resistance in sitting EOB. } \\
\text { EOB: small amplitude perturbations in all four directions. }\end{array}$ \\
\hline To initiate and train sit to stand transition & $\begin{array}{l}\text { Incorporated as per the biomechanical principles and facilitatory techniques } \\
\text { of NDT. }\end{array}$ \\
\hline $\begin{array}{l}\text { To impart lower limb weight bearing and proprioception } \\
\text { in standing }\end{array}$ & $\begin{array}{l}\text { Supported standing holding onto a walker for } 5 \text { min, keys points of control at } \\
\text { bilateral hips and knees. }\end{array}$ \\
\hline \multicolumn{2}{|l|}{ Week 4} \\
\hline Promote muscle strength & Progression with $1 / 2 \mathrm{~kg}$ weight cuffs for UL/LL muscles in mass functional patterns. \\
\hline To improve limb coordination & $\begin{array}{l}\text { Frenkel's exercises with dynamic reversals for limbs to further enhance strength } \\
\text { and coordination. }\end{array}$ \\
\hline Initiate gait training with facilitation & $\begin{array}{l}\text { Functional lower limb patterns required for gait in parallel bars with therapists } \\
\text { support and cues. }\end{array}$ \\
\hline
\end{tabular}

UL upper limb, LL lower limb, EOB edge of bed, RS rhythmic stabilization, SR stabilizing reversals, NDT neurodevelopmental technique

course of rehabilitation, there were no reports of any adverse effects, worsening of symptoms, or any other complications due to the exercise regime.

\section{Results and discussion}

Outcomes measures in this study demonstrated an improvement in overall functional performance of patient according to post-intervention scores of MMT, TCMS, and FIM (components of self-care, transfers, and locomotion). Additionally, we also observed a decline in severity of ataxia on post-test scores of SARA scale (i.e., from severe to moderate) (Tables 1 and 2).

This case is worthy of being reported because as per our knowledge, there is paucity in evidence on how individualized physiotherapy interventions involving PNF and Frenkel's coordination exercises affect functional outcomes in CAPOS syndrome. In the following 4 weeks, outcomes of present study showed a beneficial effect in overall functional status of the patient.
Key tenants for improving postural control are facilitation and muscle activation which produces purposeful coordinated movements required to carry out ADL's efficiently. PNF utilizes diagonal and rotational movement patterns to facilitate, strengthen, and promote motor control thereby producing mass functional movement patterns. It is based on the principle that control of motion proceeds from proximal to distal body regions. Facilitation of trunk control, therefore, is used to influence the extremities. PNF techniques such as dynamic reversals (slow reversals) bring about coordinated patterns between opposite muscle groups in a controlled manner. It utilizes isotonic contractions of first agonists, and then antagonists performed against resistance. Contraction of stronger pattern is selected first with progression to weaker pattern. The movements are coupled with principles of Frenkel's exercises to promote coordination. The limb is moved through full range of motion. Rhythmic stabilization (RS) and stabilization reversals (SR) help in 
proximal/larger muscles recruitment thereby bringing about postural stability in tasks such as sitting. RS is characterized by isometric contractions against the resistance provided by the therapist, with the subject trying to maintain their position as the therapist changes the direction of resistance. On the other hand, SR is characterized by alternating isotonic contractions opposed by resistance to prevent motion, allowing small movements.

Improvement seen in the TCMS scores post-intervention in our study could be attributed to enhanced trunk muscle co-activation as a result of RS and SR techniques. By the end of 4 weeks, there was considerable improvement in sitting postural control, from inability to sit at the start to being able to sit independently over edge of bed. Synofzik et al. in their study on patients with ongoing cerebellar degeneration observed improvement in multi-joint coordination and dynamic balance by intensive and continuous physiotherapy [12]. Furthermore, findings in a systematic review undertaken by Martin et al. provided modest evidence that physiotherapy may have a positive effect on gait, trunk control, and/or activity limitations of patients with cerebellar dysfunction [7].

Another important component responsible for optimal functional performance is coordination. In present study, our patient demonstrated severe incoordination in nonequilibrium tests; therefore, Frenkel's exercises were incorporated initially in supine, progressed to Fowler's position in bed followed by sitting on the edge of the bed. Exercises were aimed to improve voluntary control by sequential activation of agonist and antagonist groups of muscles using appropriate verbal and visual feedback. Principles of Frenkel's, namely perception, precision, practice, peak performance, and progression, were incorporated during the intervention. Emphasis was placed on performing the movements in diagonal PNF patterns to achieve functional movement synergies. After 4 weeks of PT protocol, we observed a reduction limb ataxia with respect to past pointing and intentional tremors. Stability at trunk is considered to be an essential factor influencing upper limb function. RS and SR coupled with dynamic reversals utilizing principles of Frenkel's exercises in functional positions could have resulted in the decrease of peripheral ataxia. Consequently, this was reflected in the post-intervention scores of self-care domain in FIM. Similar findings were observed by Pallavi et al. in her study, where intensive coordinative exercises resulted in improved motor performance and reduced ataxia symptoms, enabling patients with ataxia to achieve personally meaningful goals in everyday life [13].

Muscle weakness in bilateral extremities and trunk, coupled with ataxia was a major limiting factor for activity limitation and participation restriction in our patient. Stability of trunk serves as a key element to determine functional independence in standing and to maintain the center of mass within the base of support. RS and SR technique used in our study protocol may have helped facilitate trunk musculature and improve co-activation of abdominals and paraspinal muscles. Results of a study by Kim and Park revealed that trunk stability exercise using rhythmic stabilization could effectively enhance balance ability under one-leg and double-leg conditions. Additionally, incorporation of graded strengthening protocol with PNF techniques of slow and dynamic reversals in our protocol could have led to an improvement in strength and subsequent functional performance. Giuliani in their review report found that strength training was beneficial in improving physical performance in persons with neurological disorders [14].

The findings of this study has provided some evidence that specific rehabilitation approaches such as PNF and Frenkel's when implemented in a structured way is effective in CAPOS syndrome, thereby paving the way for future researchers to conduct studies which would give a higher level of evidence in such extremely rare disorders.

There are some limitations of this study, making it difficult to draw definite conclusions. Firstly, we focused only on one patient, who received physiotherapy in hospital, which restricts generalization. Therefore, in future, experimental studies in a larger population on patients with similar disorders could be conducted for generalizability of the protocol and better applicability. Secondly, the patient described in the paper was not followed up after the 4-week physiotherapy program, so it is not known if the effects obtained in the study persisted in the long-term observation. Lastly, our physical therapy protocol focused on impairments related to cerebellum. A more holistic/multidisciplinary approach could have been formulated involving specialists from varied fields.

\section{Conclusion}

The findings of the study provide evidence to suggest that structured and customized rehabilitation protocol can improve muscle strength, coordination and functional status and reduce severity of ataxic in an individual with CAPOS syndrome.

\section{Abbreviations \\ CAPOS: Cerebellar ataxia, areflexia, pescavus, optic atrophy, and sensorineural hearing loss; PT: Physical therapy; PNF: Proprioceptive neuromuscular facilitation; TCMS: Trunk Control Measurement Scale; SARA: Scale for the assessment and rating of ataxia; FIM: Functional independence measure scale}

\section{Acknowledgements}

We would like to extend our gratitude to IRRC committee, the participant, and all those who helped with this project. The research related to human use has been complied with all the relevant institutional policies, has followed the tenets of the Declaration of Helsinki, and has been approved by the authors' institutional review board. 


\section{Authors' contributions}

SG has contributed to the concept, design, literature search, data analysis, manuscript preparation, editing, and final review. AM has contributed to the design, literature search, data collection, and manuscript preparation. The authors have read and approved the final manuscript.

\section{Funding}

The authors received no financial support for the research, authorship, and/ or publication of this article.

\section{Availability of data and materials}

All data generated or analyzed during this study are included in this published article.

\section{Declarations}

\section{Ethics approval and consent to participate}

The protocol was approved by the local ethics committee of the MGM's College of Physiotherapy (institutional review board code MGM/COP/RRC/ 132/2019). The patient in the study was given a detailed explanation of the purpose and the procedure in the language best understood by them. Written consent was taken from patient in the language understood by him before commencing the protocol.

\section{Consent for publication}

Written informed consent to publish this information was obtained from study participant and consent to the use of his details (pictures) for this study was taken

\section{Competing interests}

The authors declare that they have no competing interests.

\section{Author details}

'Mahatma Gandhi Mission's College of Physiotherapy, Vashi, Navi Mumbai 400705, India. ${ }^{2}$ Yerala Medical Trust's College of Physiotherapy, Kharghar, Navi Mumbai, India.

Received: 21 December 2020 Accepted: 6 April 2021

Published online: 28 April 2021

\section{References}

1. Nicolaides P, Appleton RE, Fryer A. Cerebellar ataxia, areflexia, pescavus, optic atrophy, and sensorineural hearing loss (CAPOS): a new syndrome. J Med Genet. 1996;33(5):419-21. https://doi.org/10.113 6/jmg.33.5.419.

2. Rosewich H, Sweney MT, DeBrosse S, Ess K, Ozelius L, et al. Research conference summary from the 2014 International Task Force on ATP1A3Related Disorders. Neurol Genet. 2017;3(2):e139. https://doi.org/10.1212/ NXG.0000000000000139.

3. Heimer G, Sadaka Y, Israelian L, Feiglin A, Ruggieri A, Marshall CR, et al. CAOS-episodic cerebellar ataxia, areflexia, optic atrophy, and sensorineural hearing loss. J Child Neurol. 2015;30(13):1749-56. https://doi.org/10.1177/ 0883073815579708

4. Maas RP, Schieving JH, Schouten M, Kamsteeg EJ, van de Warrenburg BP. The genetic homogeneity of CAPOS syndrome: four new patients with the c.2452G>A (p.Glu818Lys) mutation in the ATP1A3 gene. Pediatr Neurol. 2016;59:71-5. https://doi.org/10.1016/j.pediatrneurol.2016.02.010

5. Adler SS, Beckers D, Buck M. PNF in practice: an illustrated guide. 3rd ed. Heidelberg: Springer Medizin Verlag; 2008

6. Dionisio V, de Baptista C, Rodrigues A, et al. Is it possible to stabilize the trunk using rhythmic stabilization in the upper limb? A cross-sectional study of asymptomatic individuals. J Man Manip Ther. 2018;26(4):212-7. https:// doi.org/10.1080/10669817.2018.1467994.

7. Martin C, Tan D, Bragge P, Bialocerkowski A. Effectiveness of physiotherapy for adults with cerebellar dysfunction: a systematic review. Clin Rehabil. 2009;23(1):15-26. https://doi.org/10.1177/0269215508097853.

8. Ojoga F, Marinescu S. Physical therapy and rehabilitation for ataxic patients. Balneo Res J. 2013;4(2):81-4. https://doi.org/10.12680/balneo.2 013.1044.

9. Milne SC, Corben LA, Roberts M, Murphy A, Tai G, Georgiou-Karistianis N, et al. Can rehabilitation improve the health and well-being in Friedreich's ataxia: a randomized controlled trial? Clin Rehabil. 2018;32(5):630-43. https://doi.org/10.1177/0269215517736903.

10. Schatton C, Synofzik M, Fleszar Z, Giese MA, Schols L, Ilg W. Individualized exergame training improves postural control in advanced degenerative spinocerebellarataxia: a rater-blinded, intra-individually controlled trial. Parkinsonism Relat Disord. 2017;39:80-4.

11. Junior PRF, De Lima S, Covolo D, Scaransi NM, Do Vale CR, Donato V. Physiotherapy in kinsbourne syndrome: a case report. Physiotherapy Quarterly. 2018;26(2):28-33.

12. Synofzik M, Brotz D, Burkard S, Giese MA, Schols L. Intensive coordinative training improves motor performance in degenerative cerebellar disease. Neurology. 2009 Dec 1;73(22):1823-30.

13. Sahay $P$, Roy $D$, Das $S$ et al. Effects of intensive coordination training while walking in parallel bars with visual feedback in a case of spinocerebellar ataxia type I: a case report. Int J Health Sci Res. 2017;7(8):507-15.

14. Giuliani C. Strength training for patients with neurological disorders. Neurol Rep. 1995;19(3):29-34. https://doi.org/10.1097/01253086-199519030-00019.

\section{Publisher's Note}

Springer Nature remains neutral with regard to jurisdictional claims in published maps and institutional affiliations.

\section{Submit your manuscript to a SpringerOpen ${ }^{\circ}$ journal and benefit from:}

- Convenient online submission

- Rigorous peer review

- Open access: articles freely available online

- High visibility within the field

- Retaining the copyright to your article

Submit your next manuscript at $\boldsymbol{\nabla}$ springeropen.com 